\title{
AGS vertical beta function measurements for Run 15
}

\author{
C. Harper, L. Ahrens, H. Huang, V. Schoefer
}

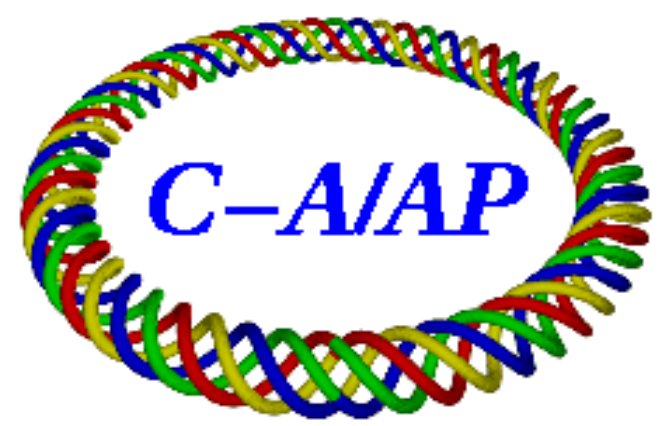

Collider-AcceleratorDepartment Brookhaven National Laboratory

Upton, NY 11973

\section{U.S. Department of Energy}

Office of Science, Office of Nuclear Physics

Notice: This document has been authorized by employees of Brookhaven Science Associates, LLC under Contract No. DE-SC0012704 with the U.S. Department of Energy. The United States Government retains a nonexclusive, paid-up, irrevocable, world-wide license to publish or reproduce the published form of this document, or allow others to do so, for United States Government purposes. 


\title{
AGS Vertical Beta Function Measurements for Run 15
}

\author{
C. Harper, L. Ahrens, H. Huang, V. Schoefer
}

October 7, 2016

\begin{abstract}
One key parameter for running the AGS efficiently is by maintaining a low emittance. To measure emittance, one needs to measure the beta function throughout the cycle. This can be done by measuring the beta function at the ionization profile monitors (IPM) in the AGS. This tech note delves into the motivation, the measurement, and some strides that were made throughout Run15.
\end{abstract}

\section{Introduction}

Knowing the transverse emittance of the beam throughout the AGS acceleration cycle is imperative for productive running conditions. Using relative measurements of the emittance, one can learn more details regarding emittance growth problems. On the other hand, taking absolute measurements gives the user insight as far as beam expectations are concerned, both upstream and downstream. Both of these measurements are needed to determine whether the emittance grows and if it does, where does this happen relative to the cycle.

In order to gain an understanding of what the emittance is, we start by measuring the width of the beam. This is translated into an emittance by knowing the beta function at the measuring instrument. Essentially, we need:

$$
\varepsilon=\sigma^{2} \beta
$$

where $\varepsilon$ is the emittance and $\beta$ is the beta function. Therefore, the knowledge of the emittance is dependent to the knowledge of the beta function.

The understanding of the beta function is particularly important for the polarized proton program. In the AGS, helical dipoles otherwise known as partial snakes are introduced to preserve the proton polarization. These partial snakes run at constant fields and generate significant optics distortion at low energies. This helical field is hard to be precisely modeled in MAD-X or ZGOUBI. Therefore measurement of beta function in AGS becomes necessary to get real beta function.

\section{Measurement Process}

We can learn the beta by distorting the equilibrium orbit and measuring the orbit motion. This was cleverly done by installing either vertical or horizontal dipoles at the respective 
IPMs. This dipole in turn puts a cusp in the closed orbit at the IPM. From there, the IPM measures the change in the profile center as the current in the corrector is varied in order to quantify the local beta function. The AGSipm application currently uses the model beta function of $22 \mathrm{~m}$ for the entire cycle.

Beam position shift $d Y$ due to a known dipole kick (with kick strength $k$ ) is given by:

$$
d Y=\frac{1}{2} k \beta\left[\frac{\cos (\pi Q)}{\sin (\pi Q)}\right]
$$

where $Q$ is the betatron tune. $\beta$ is the beta function at the point where the beta function is measured. The kick strength can be given as:

$$
k=\frac{B d l}{B \rho}
$$

where $B d l=I_{m a g} \cdot T_{m a g}$. The terms $I_{m a g}$ and $T_{m a g}$ denote the dipole current and the transfer function respectively. With this we can further simplify the equation above to get the beta function:

$$
\beta=\frac{2}{T_{\text {mag }}} \frac{d Y}{I_{\text {mag }}} \cdot B \rho \cdot \tan (\pi Q)
$$

Therefore, in order to attain this we need to measure the beam rigidity $(B \rho)$, the tune $(Q)$, and the change of the position at the IPM $(d Y)$ as we change the dipole current $\left(I_{\text {mag }}\right)$. These are all standard beam and machine parameters which would seem to imply a trivial measurement. However, it has been seen that systematic errors within these individual measurements are compounded and ultimately impact the desired goal, or the beta function.

\section{Data Acquisition and Analysis}

To perform the measurement as described theoretically in the Measurement Process section, we begin with a tune scan performed from $153-1523 \mathrm{~ms}$ in $16.5 \mathrm{~ms}$ steps. The tune scan is performed using the AGS Tunemeter application. The timing is selected such that the tune is measured in the center of each IPM integration window. The tune scan is performed before and after the beta function measurements are taken such that an average tune can be attained (Figure 1). This also accounts for small machine parameter drifts that might occur while the measurements are taking place that affect the tune.

The localized IPM dipole corrector is required to put a cusp in the closed orbit. For the vertical measurement the E15 corrector power supply is turned on with a specific function. The function for the corrector as a function of time is set through AgsOrbitControl in the Special Bump Editor Window. Although archives exist for each day a measurement was taken, typically adjustments are made to the function to ensure a maximum kick strength is generated. Again, these adjustments are necessary due to machine parameters drifting/evolving, particularly early in a polarized proton run. When analyzing the measurement data, the corrector power supply function is assumed to be $I_{\text {mag }}$. This is confirmed by monitoring the VirtualScope application to ensure the function setpoint is equivalent to the power supply readback. 

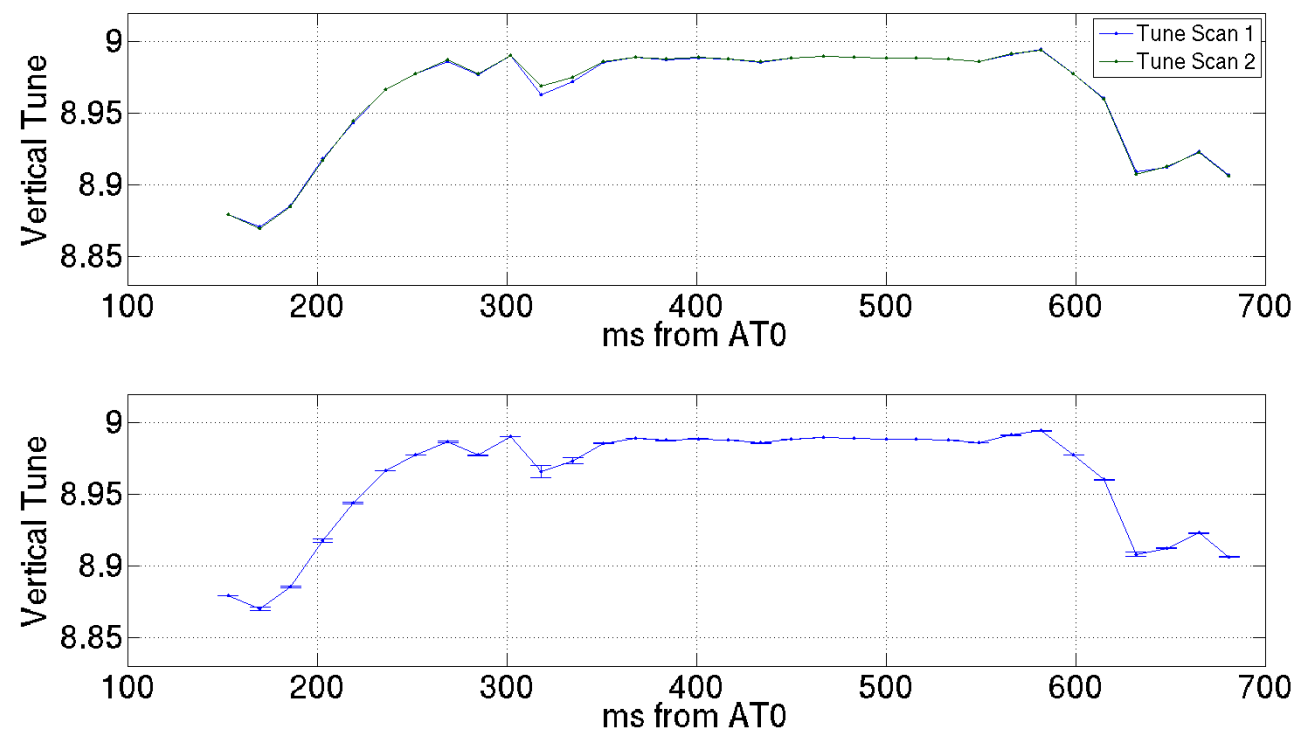

Figure 1: January 31, 2015 - Two tune scans superimposed (top) with the average and standard deviation for each time measured (bottom). Average tune is used in final beta function calculation.

The measurement process starts with the original corrector power supply function which is the maximum positive kick strength. The AGSipm application triggers and archives every AGS cycle. From here, the original function is scaled by $+\frac{1}{2}$ and the measurements are repeated. This is done for several iterations at different corrector functions. The minimum number of iterations is five and are as follows: original function, original function scaled by $+\frac{1}{2}$, original function scaled by $-\frac{1}{2}$, original function scaled by -1 , and the function scaled by 0 (no dipole kick). The IPM center data can be found in a log file and is saved over many cycles for each corrector configuration for later analysis (Figure 2). The last measured parameter used in the analysis is the beam momentum which is also saved as a log file.

A Matlab program is used for data analysis. This program loads the required log files, makes calculations based on the files loaded, and plots the relevant information in order to understand results as well as potential errors in the measurement. The first step observes the two tune scans that were taken and finds the average and standard deviation of the measurements. The average tune is the one used to calculate the betas (Figure 1). The next step plots the loaded beam centroid for each corrector setting. The beam center is plotted with respect to the time in the cycle and is averaged over many beam cycles as seen in Figure 2. The last part prior to the beta function calculation is the IPM centroid response. Here we plot the slope comparing the shift in the measured centroid motion and the relative dipole kick amplitude (Figure 3). The slope is found with these comparisons at each corrector function and the deviation of the measurement from the calculated slope is later used in the error bars for the beta function measurement. 


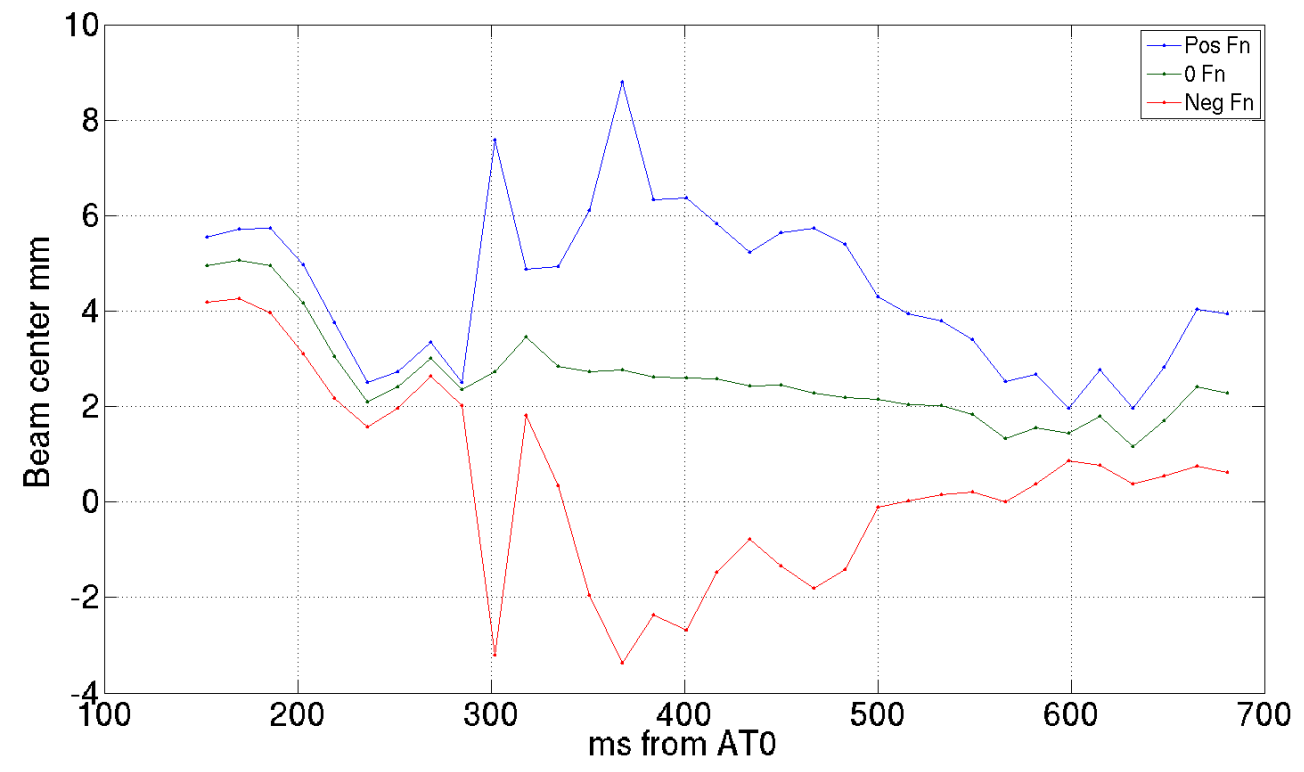

Figure 2: January 31, 2015 - Beam center position at IPM for different corrector current functions. Data points are averaged over several AGS beam cycles.
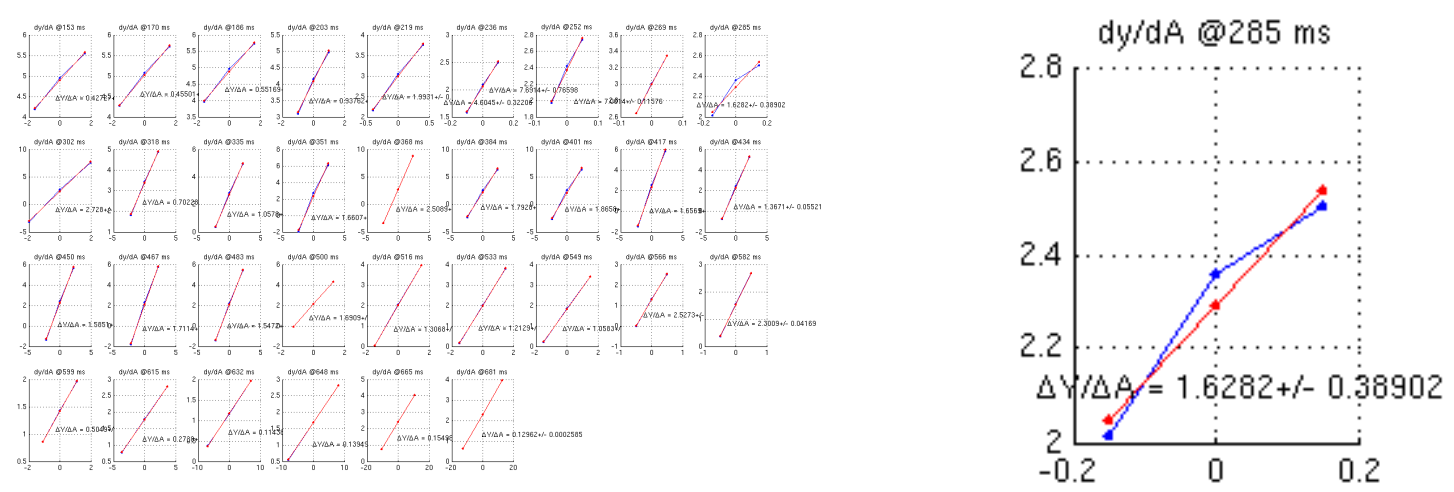

Figure 3: January 31, 2015 - IPM centroid response which plots slope comparing the shift in the measured centroid motion and the relative dipole kick amplitude. This is plotted for every measured time in the AGS cycle (left). One example can be seen for the measurement at $285 \mathrm{~ms}$ (right).

With all of these components, we have everything necessary in order to calculate the beta function. Thus far we have accounted for the beam rigidity, tune, and the change of the position at the IPM as we change the dipole current. This leads us to the last step which is to calculate the beta function which is demonstrated in Figure 4. As mentioned prior, the discrepancy between the measured vs. calculated slope plays a key role in determining the error bars for the beta function measurement. The following section delves into the analysis in the systematic errors from the data acquisition discussed here. 


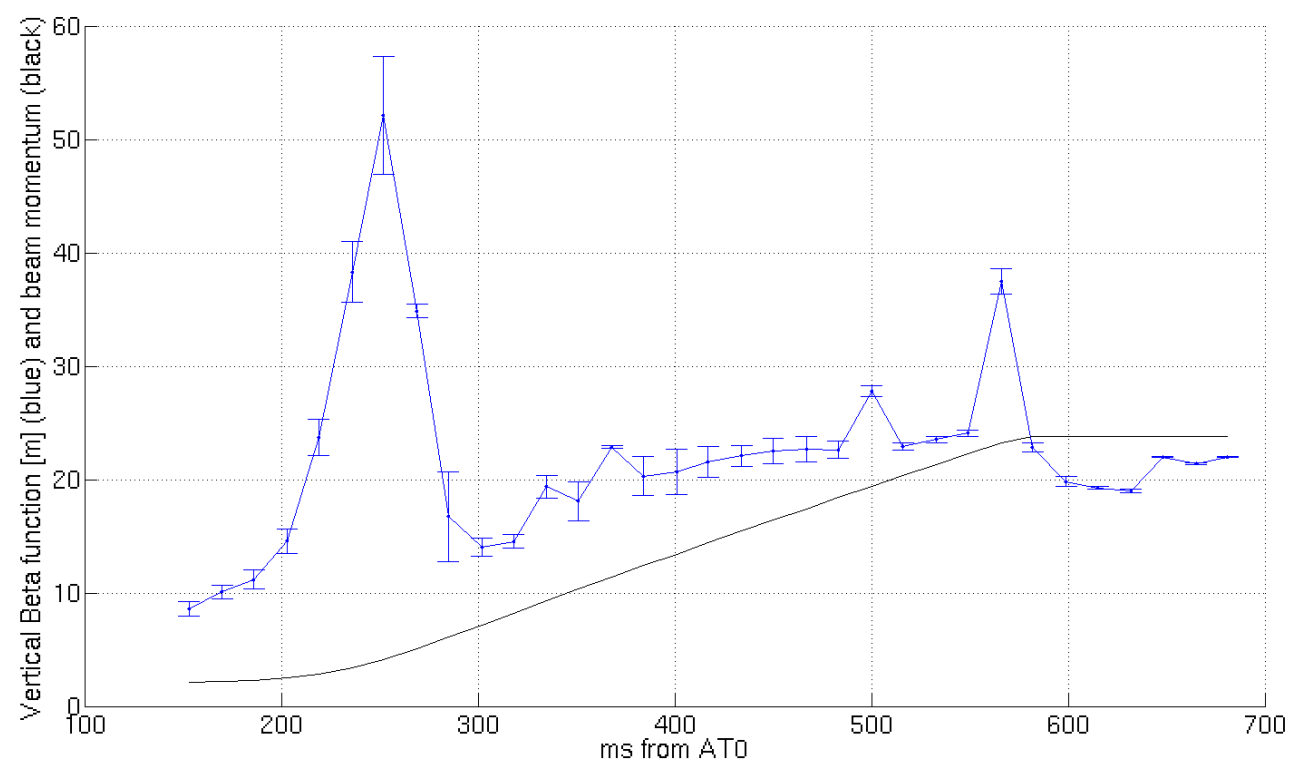

Figure 4: January 31, 2015 - Calculated vertical beta function and beam momentum.

\section{Modifications and Procedure Changes}

The primary goal for this run, in addition to measuring the beta function, was to understand compounded errors and minimize the negative impact on the beta function measurement. This was done by responding to past concerns from prior runs and exploring specific aspects of the measurement process.

\section{Hardware and Software Changes}

Since last run there were several concerns which had been addressed and have led to new implementations for Run15. The first concern was the dipole currents. As part of the beta function measurement, we need the $I_{\text {mag }}$, the dipole current. It had been assumed to be a known in that whatever the actual current function being sent was the current being produced. It was brought to our attention in Run14 that there was a significant amount of noise on the readback function for the dipole current. Over the summer, it was found that this noise from the reference and readback of the corrector current was from a common ground of both signals. To alleviate this, filters were added to these signals on all dipole correctors associated with the IPMs. Once this was done, we were able to sufficiently determine that the functions were properly being followed and the current was known. A comparison of the noisy readback signal before and after the filters were added can be seen in Figure 5.

Another concern that was discussed over the summer was the channel gains within the IPM. If these are not properly calibrated/understood, it could have a significant impact on any measurement utilized by the IPM. The channel gain calibration was done and these gains were integrated in the IPM data. It turns out, however, that in spite of this, it seems 
as though the values initially used were not far from the actual values and thus having little impact on the beta function measurements.
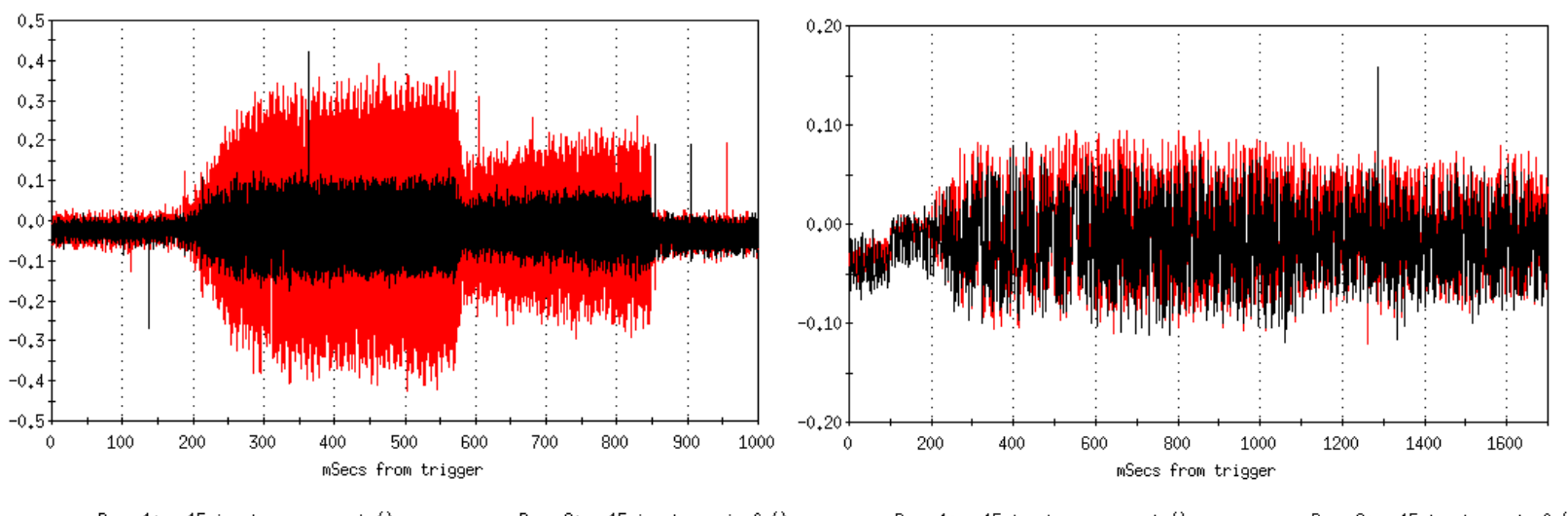

Row 1: ae15.ipm-tw-ps,current 0 Row 2: ae15.ipm-tw-ps,iref 0

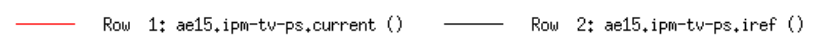

Figure 5: Comparison between two E15 corrector current function demonstrating the noise reduction from 2014 and 2015. The left image is from 2014 showing excessive noise between the reference (black) and the readback (red) and the right shows the reduced noise in 2015 after filters were added. Both images use a corrector current of ' $0 \mathrm{~A}$ '.

The final concern mentioned here is the matlab code used to generate the beta function values based upon the measured parameters. Until this run, the centroid motion was taken as an average over the whole cycle. Although it sometimes gave reasonable results, there were times where the measurements had undesirable results. Over the summer, there were changes made to the program such that each measured time in the cycle was analyzed individually. Therefore, special care was taken to understand how the centroid was moving with regards to its time in the AGS cycle. This was proved to be more accurate and consistent with what the model deemed to be correct.

With these improvements, it was found early on that the beta function could be measured reliably and consistently. Therefore, the majority of the run was dedicated to look for unknown pitfalls and make the measurement more robust to compounded flaws.

\section{Vacuum Pressure Effect}

A critical part of the beta function measurement is the usage of the AGS IPM. The AGS IPM measures the ions produced as a result of the circulating beam passing through gas in the vacuum chamber. The number of ions produced from collisions with the circulating beam is a function of the beam current and the density of the gas. When using protons, the number of ions generated from collisions with residual gas is too small so to increase the signal size, a controlled leak of $\mathrm{CO}_{2}$ is used to 'spoil' the vacuum. (This does not apply to heavy ions since there are generally enough ions produced to measure a good signal-this paper only focuses on proton beam). 
Clearly, the vacuum is paramount in generating a proper signal for our measurement. In the past, the vacuum was controlled such that it was at the $4 \mathrm{E}-7$ Torr level. However, there was not extensive work done recently to see if this was adequate enough for our measurement. Could our signal been distorted enough to change the final outcome?

The first iteration of this measurement was done on January $31^{\text {st }}$. There were two measurements made. The first measurement was done in the same fashion as in prior runs. The second measurement spoiled the vacuum further but kept all other parameters the same. This way all other variables were constant in theory.

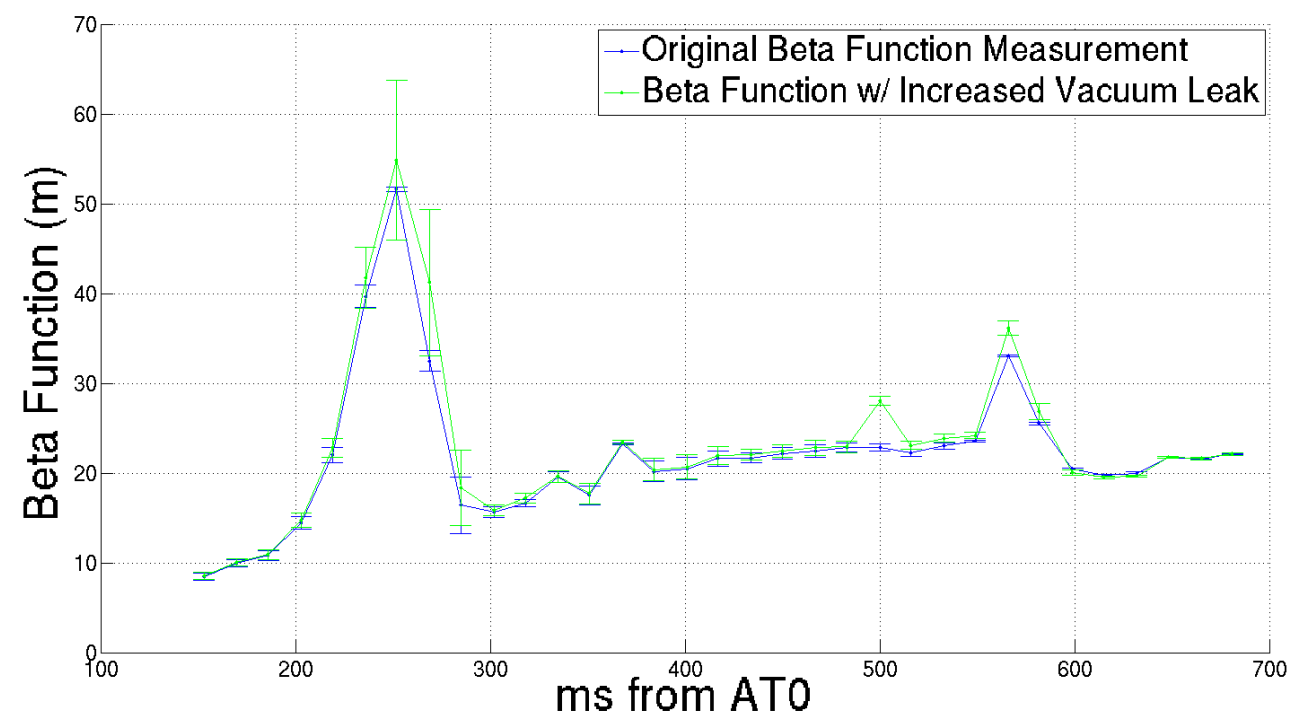

Figure 6: Beta function comparison with various vacuum pressures. Green trace indicates more 'spoiled' vacuum than blue trace. Error bars are generated from the spread of the measured beta and the fitted data (fit attained from 5 individual measurements at various dipole corrector currents.)

As seen in Figure 6, the results were not resounding. With the exception of a few points, the final beta function measurement remained essentially the same. This would in theory confirm that our typical vacuum settings are sufficient for a nominal setup for the beta function, therefore very little error can be attributed to the vacuum.

However, this run another parameter was varied-the integration window. Although the vacuum held for the default setting of an $8 \mathrm{~ms}$ integration window, this is not necessarily true for all integration window settings. This idea was further explored and will be explained further in this tech note.

\section{Tune Measurements}

In normal operation, the two kicks of the horizontal and vertical planes in the AGS tunemeter are separated by 300 turns. Depending on what plane is the focus, the trigger timing of the tunemeter is varied accordingly. Early in the run there was an instance where the 
tunemeter was setup to trigger on a time more relevant for the opposite plane of interest for the beta function measurement. In this instance the measurement took place 300 turns after the desired trigger time, which amounts to approximately a $0.8 \mathrm{~ms}$ delay. This brought about a relevant question: how sensitive is the tune measurement and its impact on the beta function results? This is particularly pertinent when the tunes are driven close to the integer.

Last year, Leif had given a presentation which further simulated the dependence on the tune with relation to the betatron fractional error. He found that in order to satisfy our quality 'benchmark' of $5 \%$ we need a tune error of less than 0.005 for tunes between 8.6 and 8.9. This is easily achievable. However, as the tunes approach 9, we need to be an order of magnitude better (0.0005) in tune error to still achieve our goal of $5 \%$. This still is possible but this becomes increasingly difficult to achieve and one must start to become concerned about stability. Therefore, with all of this in mind, a .8ms shift in time could potentially be disastrous for our measurement, due to a significant part of the AGS cycle having a vertical tune above 8.95 .
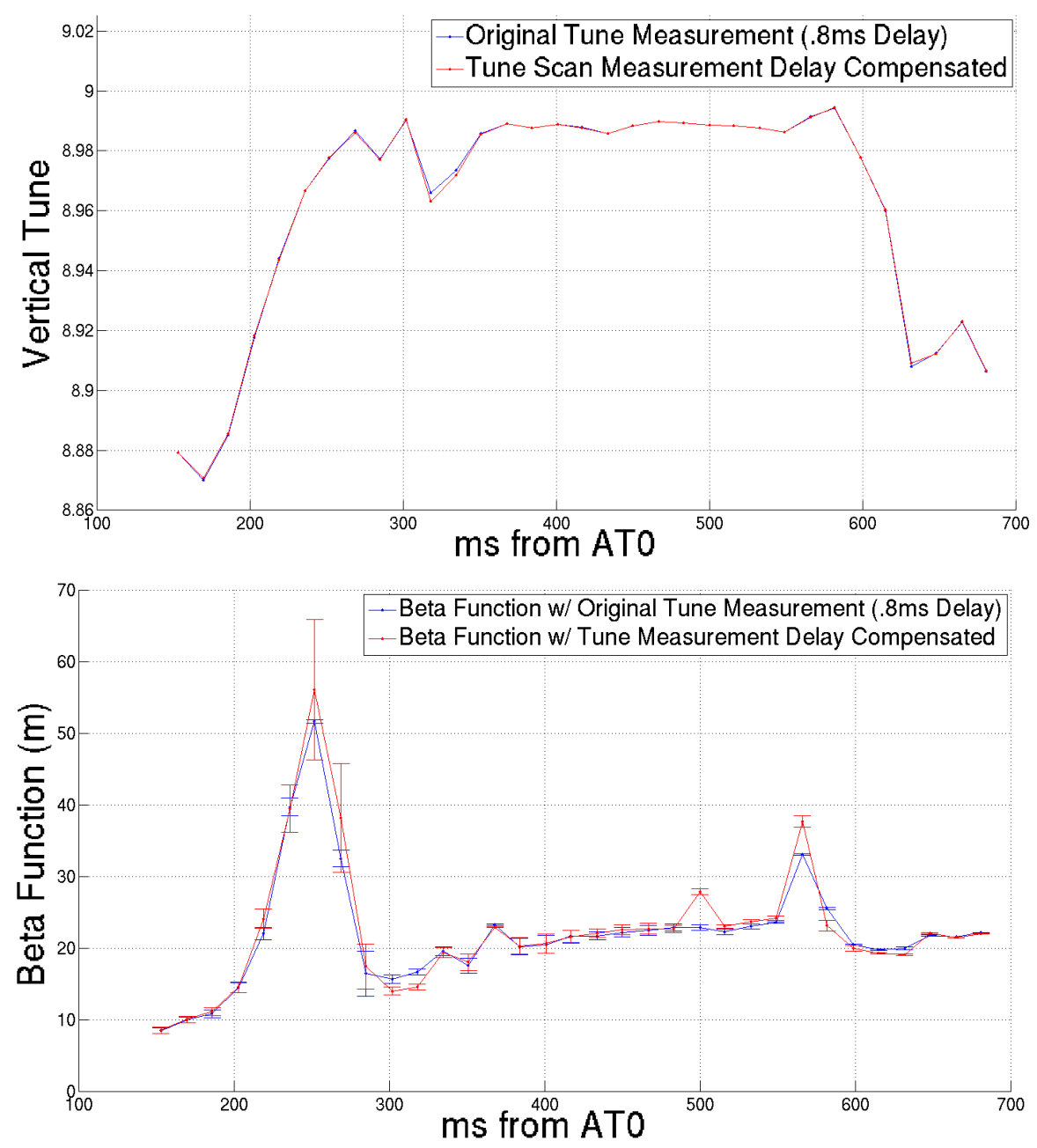

Figure 7: Tune comparison with tunemeter delay (top) and resulting beta function(bottom). 
The first step was to try and recreate the original findings and compare to see what happens to the measurement. On January 31st, a beta function measurement was taken. However, there were two different tune scans taken that day-one with the correct tunemeter settings and another with the $.8 \mathrm{~ms}$ delay. All other parameters were kept constant. The results are shown in Figure $\%$.

As indicated in Figure 7, the results are seemingly subtle, but are relevant. When the tunes are high or quickly changing, it seems as though our measurement is more susceptible to incorrect tunes and thus an incorrect beta function measurement. A seemingly small error propagates to become a larger one at the final result.

This leads us to another concern with regards to the tune over the integration window of the IPM. The IPM takes data over an integration window that the user indicates from the AGSipm application. The integration window is typically set to its default setting of $8 \mathrm{~ms}$, but the user has the ability to change it from the AGSipm application under Setup $\backslash$ Timing $\backslash$ Simple $\backslash$ Integration Period (ms). If the tune is as sensitive over a .8ms then a window of $8 \mathrm{~ms}$ can have a huge impact. Particularly at high tunes or quickly changing tunes we may not have an accurate depiction of the tune measurement for larger integration windows.
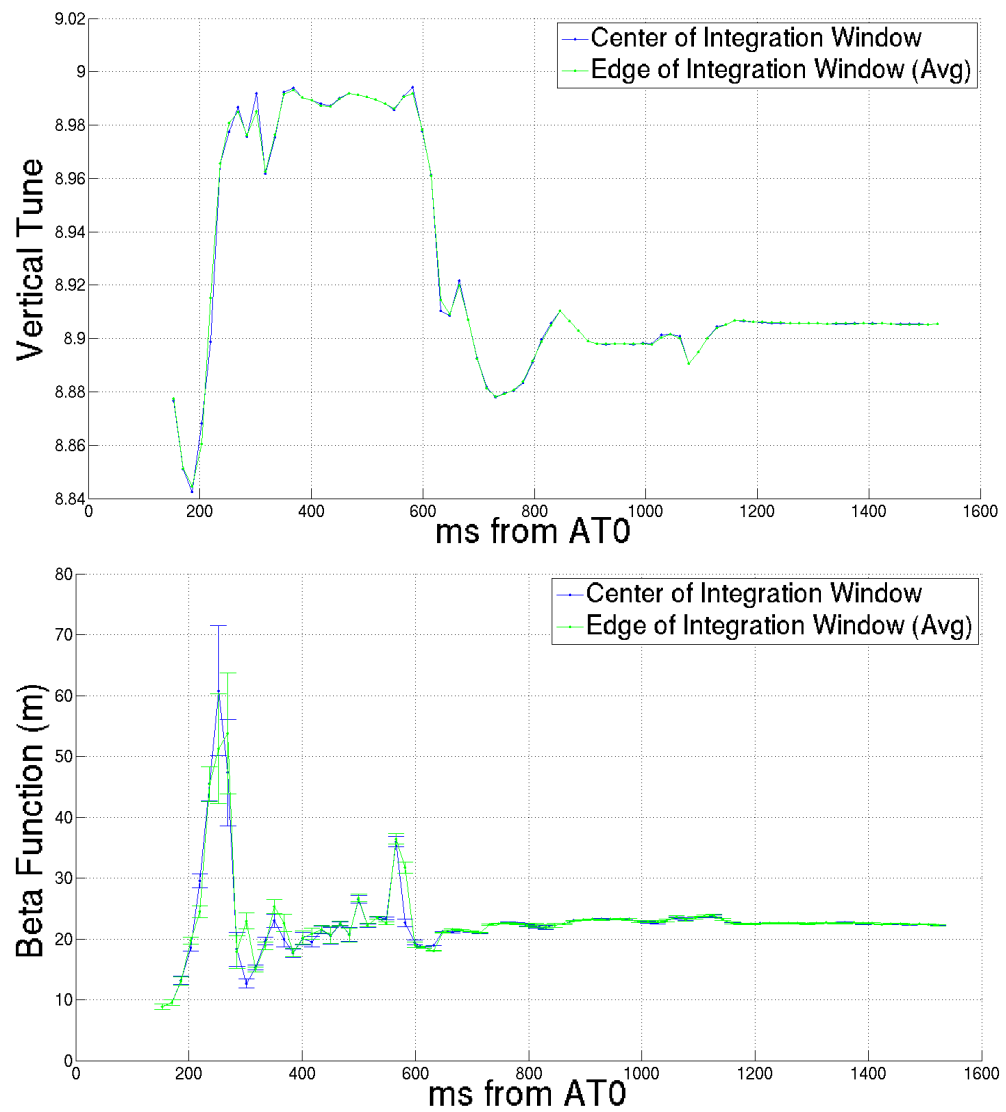

Figure 8: Tune comparison when measuring the tune at various points in the integration window (top) and resulting beta function(bottom). 
We further studied this idea over several iterations. The focus was to get a more accurate representation of the tune. Therefore, there were a minimum of three tune scans that were taken. The first was the same as in prior runs in that the tune measurement was taken in the center of the integration window. (Typically, two of these scans were done and an average of the tunes was taken as mentioned earlier.) The second and third measurements were taken on the edge of the integration window. Thus, for example, an $8 \mathrm{~ms}$ integration window would have the tune measured in the center of the window and $4 \mathrm{~ms}$ earlier and later from that point. From there, the tunes measured at the edges are averaged. It is clear that the tunes measured at the edge needed to be adjusted depending on the set integration window. In Figure 8 we see an example of the tunes measured in the center of the integration window in comparison to the average tune as it was measured at the edge of the integration window.
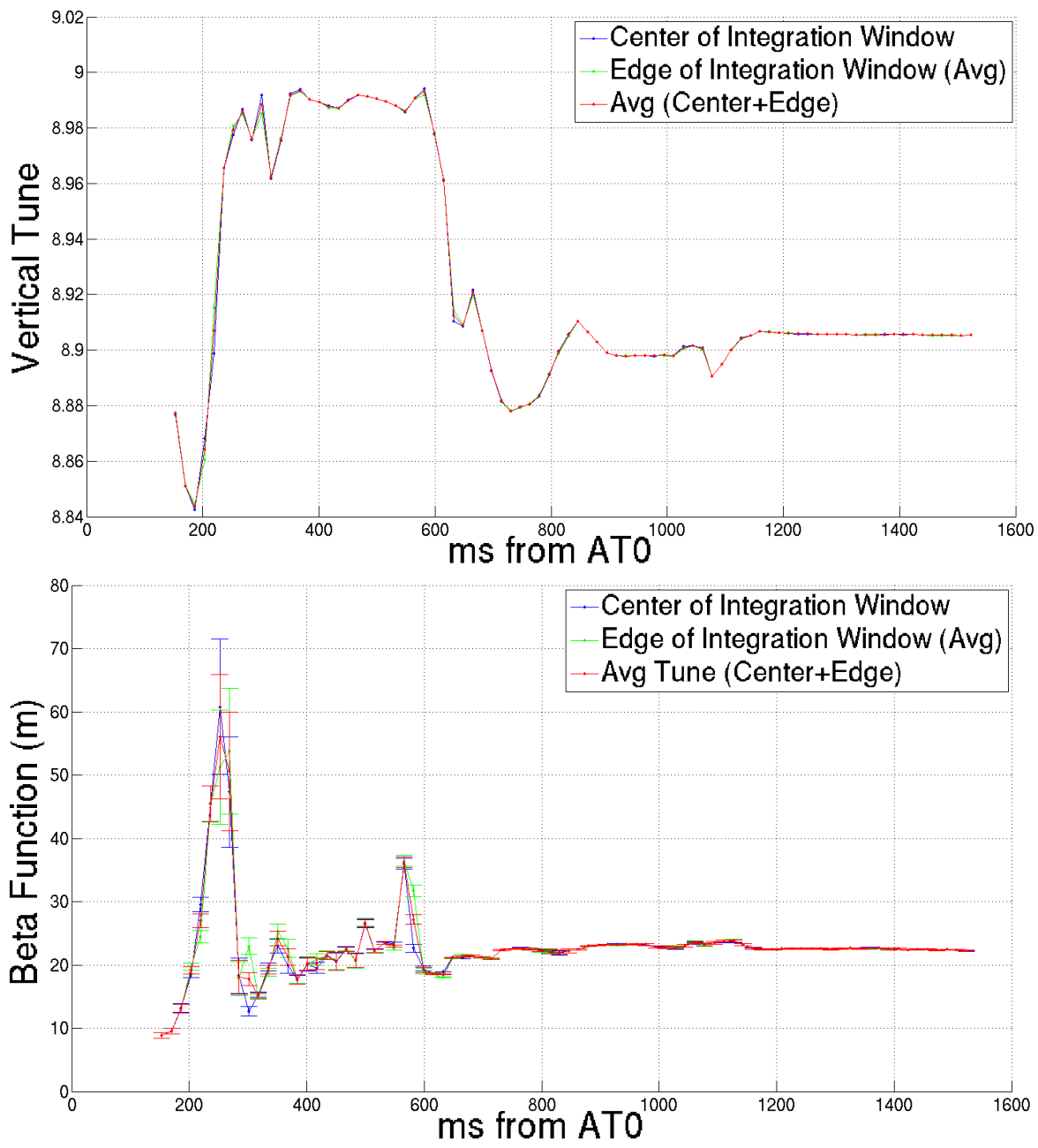

Figure 9: Tune comparison when measuring the tune at various points in the integration window (above) and resulting beta function(below). 
In the end it was determined that this difference in the timing for the tune measurement was the most accurate representation of the machine. The tune measured at the center of the window and the average of the tunes measured at the edge were averaged together to generate the tunes used for the beta function measurement. This way, the tunes over the entire integration window were considered. However, the tune measurements are not weighed the same- by using the central tune and the average of the tunes measured at each edge, there is more of an emphasis on the tune measured at the center. That is, the tune measured at the center is weighted twice as much as each of the edge tunes that contribute to the averaged tune. However, as mentioned before, this is all dependent on the size of the integration window.

In theory by limiting the size of the window, the tunes should be more accurate and generally less fluctuations. In order to truly come to those conclusions we needed to get a more thorough understanding of the integration window in general and the impact it actually has on the beta function measurement.

\section{Integration Window}

So up until now we have looked at specific aspects of the beta function measurement. However by looking into these nuances we know that these aspects are further influenced by the integration window. The integration window, otherwise known as the integration period, is the duration that the analog integrators in the IPM integrate the charge. Therefore, the longer the integration period, the more fluctuations could take place during the measurement. Thus it is clear that we need to find the optimal setting in order to capitalize on all aspects of the beta function measurement.

The first instance of this was looking at the impact the window had on the tune measurement. As mentioned earlier, we had seen that the tune measurement is impacted at high tunes and areas where the tune changes quickly. This becomes especially true with longer integration periods. Therefore, as an attempt to combat this, three tune measurements are used for each beta function measurement. The first measurement is in the center of the integration window and the other two are at the edge of the integration window which in turn is averaged. Obviously, depending upon the integration period size, the tune measurement times would need to be adjusted. Ideally, the tunes should remain constant regardless of when they were actually measured. The difference in tune measurements can be observed in Figure 10.

In theory, the shorter the integration window, the more accurate the measurement ought to be. Since the period in which the charge is being integrated is reduced there is less room for fluctuation in the measurement to occur. However, it was found during the first iteration of this that as the integration window got smaller, the worse the measurement got at the end. It was not initially clear as to why this would be until a closer look was done for the signal on the IPM. Using Logview data, we could see the position of the centroid for each of the corrector current setpoints. When comparing these centroids for each of the integration 

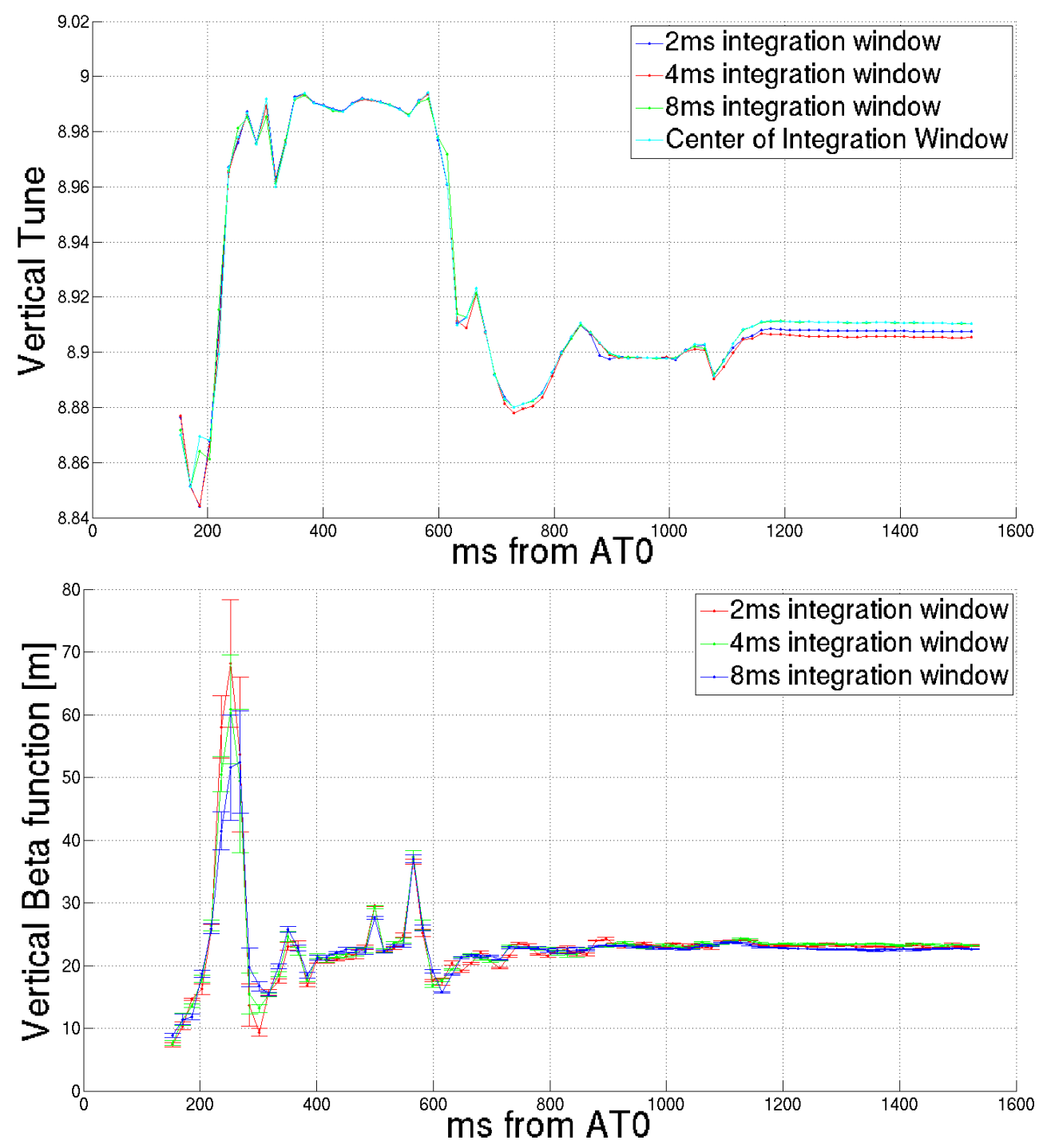

Figure 10: Tune comparison with various integration window lengths (top) and resulting beta function (bottom).

window settings, there seemed to be a 'jitter' that got progressively worse the shorter the period became. It was perhaps due to the vacuum not being adjusted in accordance with the change in the integration window. In spite of the prior findings (mentioned in Vacuum Pressure Effect section), perhaps the vacuum did have an impact on the final measurement, it just was not clear without the shorter integration window.

This led to the final measurement where the vacuum was varied at three independent integration window timings: $8 \mathrm{~ms}, 4 \mathrm{~ms}$, and $2 \mathrm{~ms}$. For each integration window timing three distinct setpoints were used for the $\mathrm{CO}_{2}$ gas leak: 1100, 1200, and 1300. These settings have an average vacuum pressure of $2.5 \mathrm{E}-7$ Torr, $3.5 \mathrm{E}-7$ Torr, and 4.7E-7 Torr respectively. The results for select measurements is seen in Figure 11. 

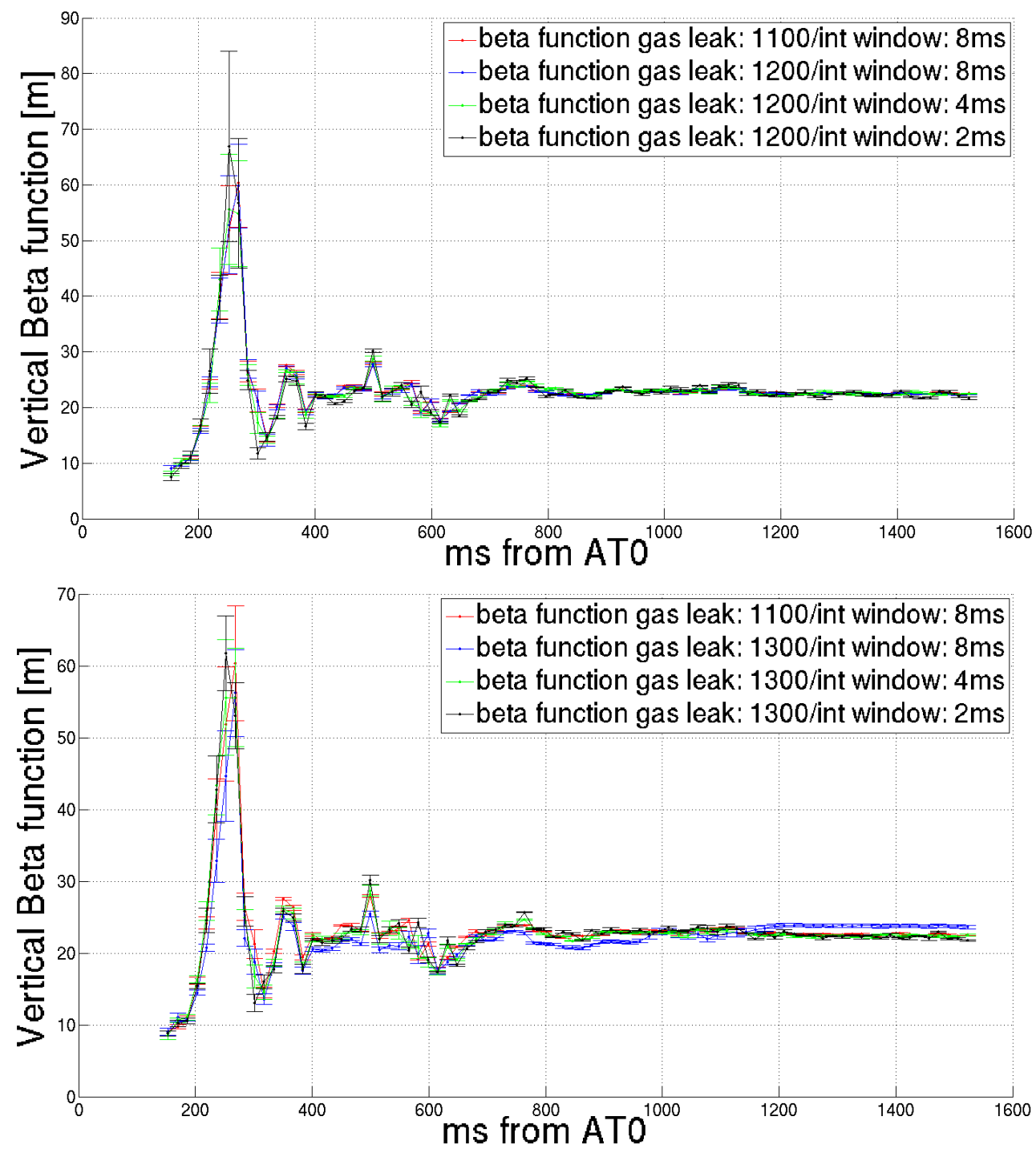

Figure 11: Beta function comparison with various vacuum pressures and integration windows. Bottom figure has more 'spoiled' vacuum than top figure.

The conclusion is that the longer integration window requires less of the $\mathrm{CO}_{2}$ leak whereas the shorter integration window requires more $\mathrm{CO}_{2}$ leak (ie more spoiled vacuum). This can be seen when comparing the integration window with various vacuum settings, the beta functions tend to agree less and less as the leak gets higher for $8 \mathrm{~ms}$, yet better with the $2 \mathrm{~ms}$. This cannot be said with concrete evidence however, and more measurements would need to be taken in the future where the vacuum is spoiled further. As of now, it would appear as though any integration window is valid for this measurement as long as the vacuum pressure was adequate. At this time, the longer integration window is the most stable due to better control of the vacuum pressure. In the future, with better control of the $\mathrm{CO}_{2}$ leak, a shorter integration window should be utilized.

With regards to pursuing information on this-after talking with vacuum group personnel, 
it seems as though there can be some room as far as the vacuum pressure in concerned. The vacuum valves will close when the pressure goes higher than 5E-6 Torr or if two of the three ion pumps in the loop trip off. Based on logged data, it appears as though ion pumps were running whenever the vacuum leak was increased, so this may be the limiting factor.

\section{Looking Forward}

Although a lot of ground was covered over Run15, there is still plenty of room for improvement. Everything that has been mentioned thus far has taken place with the vertical ion IPM located at E15. In the future we hope to expand our knowledge of local beta function measurements at other IPMs. In this section we delve into the various other areas where improvement can be made.

\section{Horizontal Ion IPM}

To date, there has yet to be a successful horizontal beta function measurement using the ion IPM at C05 for the entire duration of the AGS cycle. The only section of the cycle that has been successfully completed is at flattop. It was discovered in Run14 that despite the deliberate attempt to move the orbit at injection or up the ramp at C05, it seemed as though the orbit held steady. This is due to the corrector's close proximity to the RF PUE's at sections B18 and C12. Since the radial loop is used early on in the AGS cycle, whenever a distortion was made in the orbit between these PUE's, the average orbit would be moved to compensate for these changes. This unfortunately would distort any measurement that was made.

However, while at flattop, the phase loop is used and the distortions made in the orbit by $\mathrm{C} 05$ were unperturbed. Therefore adequate measurements were made and thus far have agreed with the model. However, it would be interesting to get data somewhere other than at flattop.

There has been some discussion with regards to getting information about the beta function up the ramp. It would be possible to work with LLRF personnel in order to find a workaround. It seems unlikely that a whole cycle can be attained like it has for E15. The idea is that the phase loop could be used for certain parts of the cycle that are not necessarily typical, however this may not be possible in certain areas (ie transition). This will need to be further explored in subsequent runs.

\section{eIPMs}

There is a horizontal eIPM at D05 and a vertical eIPM at D15. With regards to the beta function measurements, little work was done with the eIPM's this run. Although results were capable to be generated, there is little agreement between various measurements or even the model. Predominantly, this is attributed to this being a new system which was still being commissioned-it may not have been configured correctly quite yet. 
During the shutdown between Run14 and Run15, there was work done on the dipole corrector power supplies for both D05 and D15. After the upgrades the supplies were capable of ramping from $0 \mathrm{~A}$ to $12 \mathrm{~A}$ in $35 \mathrm{~ms}$, whereas in Run14, the same thing would require 100ms. In addition, adjustments were made such that the maximum current attainable was 20A. This held true for functions loaded for D15, however, there were several instances where D05 dipole corrector would trip due to the current function being too steep, in spite of the fact that it followed the power supply specifications.

The burning question is why the measurements did not agree, neither with each other or with the model. There are currently some theories which give us some insight. One thought is that the transfer function for the power supplies is not correct. Although this would not explain why the measurements don't agree, it would explain how nothing thus far agrees with the model, and the fact that current measurements are approximately a factor of two too large. In addition, channel gains had not been calibrated prior to most of the measurements. We are particularly sensitive to this and, again, this does not explain the discrepancy in measurements, it is another contributing factor when comparing this to model data. With regards to the different measurements it appears that all aspects of the measurements are equivalent except for the corrector currents (and thus the orbit). Repeatability should be a goal for subsequent runs to further diagnose this particular issue.

\section{Conclusion}

For the duration of Run15 the vertical beta function at E15 IPM was extensively measured. During this time several parameters were scanned. The vacuum leak and integration window was varied for the beta function measurement. In the range we changed these parameters, the beta function results did not change much. Nevertheless, a shorter integration window with higher gas leak seems to be the natural choice. The effect of the betatron tune during the integration window was also studied. It is important, and could have significant effect on the beta function measurement. The average of several tune measurements along the integration window seems to be the valid one. In Figure 12, we demonstrate the optimized measurement and compared the results with the AGS model. In Table 1, a detailed look of each individual point is demonstrated. Going forward, there will be dedicated time to understand the horizontal beta function (C05) and the use of eIPMs (D05 and D15). 

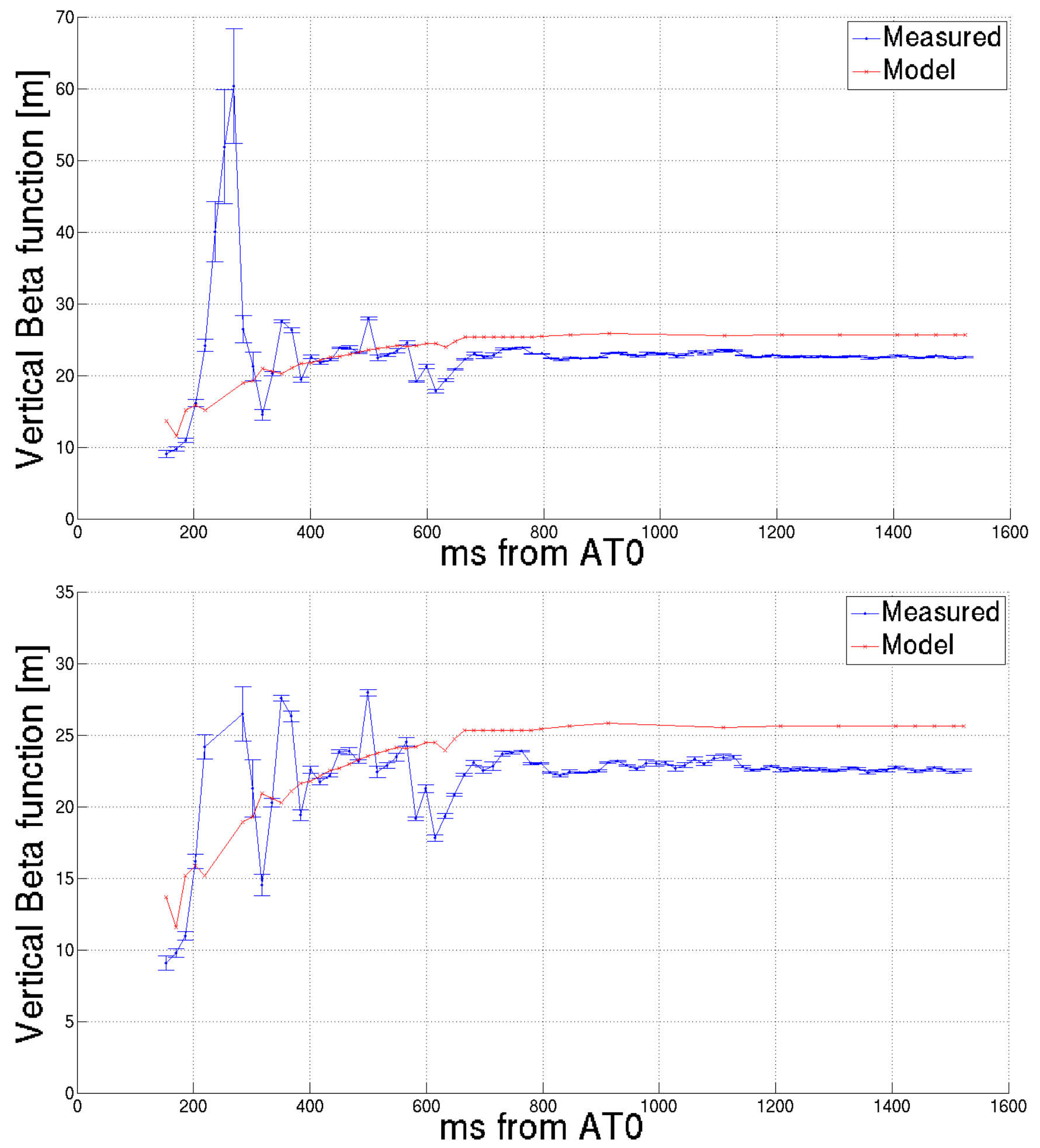

Figure 12: Beta function comparison between data taken from April 9th and the model. Top one includes points at $0+$, bottom one does not-the model does not include these points as they do not converge. 
Table 1: Detailed measurements for each point as gathered from Figure 12.

\begin{tabular}{|l|l|l|l|l|l|l|}
\hline $\begin{array}{l}\text { Time } \\
(\mathbf{m} \text { ) from } \\
\text { AT0) }\end{array}$ & Beta & Error & & $\begin{array}{l}\text { Time } \\
(\mathbf{m s} \text { from } \\
\text { AT0) }\end{array}$ & Beta & Error \\
\hline 153 & 9.075 & 0.508 & & 846 & 22.442 & 0.118 \\
\hline 170 & 9.752 & 0.298 & & 863 & 22.370 & 0.058 \\
\hline 186 & 10.980 & 0.297 & & 879 & 22.402 & 0.045 \\
\hline 203 & 16.187 & 0.488 & & 896 & 22.502 & 0.085 \\
\hline 219 & 24.176 & 0.864 & & 912 & 23.069 & 0.069 \\
\hline 236 & 40.062 & 4.186 & & 929 & 23.162 & 0.037 \\
\hline 252 & 51.879 & 7.931 & & 945 & 22.884 & 0.052 \\
\hline 269 & 60.346 & 7.959 & & 962 & 22.630 & 0.113 \\
\hline 285 & 26.487 & 1.890 & & 978 & 23.047 & 0.228 \\
\hline 302 & 21.271 & 2.018 & & 995 & 23.001 & 0.166 \\
\hline 318 & 14.524 & 0.752 & & 1011 & 23.069 & 0.130 \\
\hline 335 & 20.264 & 0.313 & & 1028 & 22.670 & 0.193 \\
\hline 351 & 27.570 & 0.191 & & 1044 & 22.893 & 0.174 \\
\hline 368 & 26.309 & 0.380 & & 1061 & 23.330 & 0.123 \\
\hline 384 & 19.410 & 0.367 & & 1077 & 22.977 & 0.102 \\
\hline 401 & 22.563 & 0.253 & & 1094 & 23.395 & 0.176 \\
\hline 417 & 21.742 & 0.213 & & 1110 & 23.446 & 0.225 \\
\hline 434 & 22.198 & 0.144 & & 1127 & 23.467 & 0.132 \\
\hline 450 & 23.846 & 0.116 & & 1143 & 22.792 & 0.075 \\
\hline 467 & 23.862 & 0.259 & & 1160 & 22.547 & 0.069 \\
\hline 483 & 23.158 & 0.114 & & 1176 & 22.622 & 0.027 \\
\hline 500 & 27.951 & 0.229 & & 1193 & 22.819 & 0.057 \\
\hline 516 & 22.428 & 0.398 & & 1209 & 22.575 & 0.134 \\
\hline 533 & 22.893 & 0.197 & & 1226 & 22.560 & 0.099 \\
\hline 549 & 23.459 & 0.276 & & 1242 & 22.634 & 0.119 \\
\hline 566 & 24.522 & 0.311 & & 1259 & 22.592 & 0.113 \\
\hline 582 & 19.162 & 0.134 & & 1275 & 22.634 & 0.113 \\
\hline 599 & 21.266 & 0.270 & & 1292 & 22.524 & 0.102 \\
\hline 615 & 17.800 & 0.243 & & 1308 & 22.540 & 0.084 \\
\hline 632 & 19.333 & 0.177 & & 1325 & 22.680 & 0.109 \\
\hline 648 & 20.814 & 0.093 & & 1341 & 22.663 & 0.076 \\
\hline 665 & 22.221 & 0.102 & & 1358 & 22.387 & 0.115 \\
\hline 681 & 23.053 & 0.181 & & 1374 & 22.490 & 0.081 \\
\hline 698 & 22.549 & 0.233 & & 1391 & 22.519 & 0.085 \\
\hline 714 & 22.821 & 0.297 & & 1407 & 22.750 & 0.087 \\
\hline 731 & 23.686 & 0.180 & & 1424 & 22.639 & 0.056 \\
\hline 747 & 23.755 & 0.064 & & 1440 & 22.484 & 0.110 \\
\hline 764 & 23.893 & 0.048 & & 1457 & 22.521 & 0.062 \\
\hline 780 & 23.006 & 0.071 & & 1473 & 22.743 & 0.048 \\
\hline 797 & 23.008 & 0.053 & & 1490 & 22.546 & 0.089 \\
\hline 813 & 22.374 & 0.061 & & 1506 & 22.378 & 0.074 \\
\hline 830 & 22.155 & 0.093 & & 1523 & 22.530 & 0.076 \\
\hline & & & & & & \\
\hline & & & & & & \\
\hline
\end{tabular}




\section{References}

[1] H. Weisberg et al., Proc. of PAC'83, p.2179.

[2] H. Huang et al., Proc. of IBIC2013, p.492.

[3] R. Connolly, et al., Proc. of IBIC2014, p.39. 